\title{
Magnetohydrodynamic Dissipation
}

\author{
L. Biermann and A. Schlüter \\ Max Planck Institut für Physik, Böttingerstrasse 4, Göttingen, Germany
}

$\mathrm{B}^{\mathrm{E}}$ EFORE entering the subject of magnetohydrodynamic dissipation we comment briefly on the question of the supply of kinetic energy to the interstellar gas. This topic has been considered in the 1953 Symposium by Schlüter and myself, ${ }^{1}$ and also by Oort. As had been pointed out already by Spitzer in Paris, 1949, the visible HII regions, owing to their excess pressure as compared with the $\mathrm{HI}$ regions and the dilute HII regions, must be assumed to expand with a velocity of the order of 10 or $20 \mathrm{~km} / \mathrm{sec}$. By this expansion part of the radiation energy of the star is converted into kinetic energy. It was estimated (p. 153 of the proceedings $s^{1}$ ) that a typical $\mathrm{HII}$ region around a $\mathrm{BO}$ star feeds $10^{35} \mathrm{ergs} / \mathrm{sec}$ to the instellar gas, and that the number of these regions is such, that each region has to provide kinetic energy on the average to $10^{36}-10^{37} \mathrm{~g}$ of interstellar material. Thus a value of $10^{-2}-10^{-1}$ erg $\mathrm{g}^{-1} \mathrm{sec}^{-1}$ was found (which corresponds to $10^{-26}-10^{-25}$ erg $\mathrm{cm}^{-3} \mathrm{sec}^{-1}$, assuming $10^{-24} \mathrm{~g} / \mathrm{cm}^{3}$ for the mean density of the interstellar material in the disk).

In this paper, we discuss three different mechanisms by which this supply of kinetic energy of the gas in interstellar space or in the vicinity of stars may be dissipated and ultimately converted into heat. For all of these mechanisms it is of decisive importance that the cosmic gas is at least partly ionized and is almost everywhere pervaded by magnetic fields.

The first process results from the fact that in a partially ionized gas only the ionized component interacts with the magnetic field, whereas the neutral component does not. Hence the volume forces, exerted by the magnetic field, act only on the ionized component of the gas, leading thus to a differential velocity of the plasma relative to the non-ionized component. This process may be called ambipolar diffusion, using a term which has been adopted in the theory of gaseous discharges.

The dissipation by ambipolar diffusion is most effective in $\mathrm{HI}$ regions, for which it has been discussed seven years ago by Schlüter and myself ${ }^{2}$ and more recently by Cowling. ${ }^{3}$ Denoting the cross section for collision between charged and neutral particles by $q\left(\approx 10^{-15} \mathrm{~cm}^{2}\right)$, and the number densities of the neutral particles and the ions by $N_{0}$ and $N_{i}$, respectively (of the order of $10^{+1}, 10^{-2} \mathrm{~cm}^{-3}$, respectively), the diffusion velocity by $\Delta v$ and the thermal velocity by $v_{\text {th }}$, the

\footnotetext{
${ }^{1}$ Gas Dynamics of Cosmic Clouds, Amsterdam 1955, p. 144.

2 A. Schlüter and L. Biermann, Z. Naturforsch. 5a, 237 (1950)

3 T. G. Cowling, Monthly Notices Roy. Astron. Soc. 116, No. 1 (1956).
}

collision frequency will be either $\Delta v N_{0} N_{i} q$ or $v_{\text {th }} N_{0} N_{i} q$, whichever is larger, and since the loss of momentum per collision is $m_{H} \Delta v$, the force necessary to maintain the diffusion velocity is of the order $\left(m_{H} \Delta v\right) \cdot\left(\Delta v N_{0} N_{i} q\right)$. Here we assume that $\Delta v$ is at least comparable with the thermal velocity, as we shall find it to be. If we use $L$ as the length scale for the magnetic field, the driving force will be of the order $B^{2} /(4 \pi L)$. Hence we arrive at

$$
(\Delta v)^{2} \cong B^{2} \lambda / 4 \pi \rho L,
$$

where $\rho=\left(N_{0}+N_{i}\right) m_{H}$ and $\lambda=1 / N_{i} q$, the mean free path for a neutral atom.

Using numbers which may be appropriate for an $\mathrm{HI}$ region $\left(\lambda \approx 10^{17} \mathrm{~cm} ; L \approx 10^{20} \mathrm{~cm}\right)$, we obtain

$$
\Delta v \approx 10^{4} \cdots 10^{5} \mathrm{~cm} \mathrm{sec}^{-1} \text {. }
$$

The energy dissipated per collision is $\left(\frac{1}{2}\right) m_{H}(\Delta v)^{2} \sim 10^{-15}$ erg. Thus the total energy dissipated is $10^{-4} \cdot 10^{-3} \mathrm{erg}$ $\mathrm{g}^{-1} \mathrm{sec}^{-1}$. The result shows that the energy dissipated by ambipolar diffusion in HI regions is probably smaller than the energy dissipated by turbulence and viscosity, but not by very much. If shock fronts dominate the ordinary dissipation of kinetic energy, then between the shock fronts $\Delta v$ will be smaller than assumed, but within the fronts $\Delta v$ will be of the order of the velocity of the shock front itself. Owing to the relatively small number of non-ionized atoms, the energy dissipated by ambipolar diffusion will again be smaller, but not very small compared with that dissipated by viscosity. Ambipolar diffusion will however influence the shape of the shock fronts.

In dense HII regions we may have $1 \%$ non-ionized atoms which will move essentially as the ions do. Using the same approach as in the former case the dissipated energy is now found to be insignificant because two factors enter which are both small compared with unity: first, the ratio (mean free path of an ion for collision with a neutral atom)/(scale length of the magnetic field); and second, $N_{0} / N_{i}$.

In dilute HII regions between clouds in spiral arms, or between the spiral arms themselves, the relative contribution of ambipolar diffusion is also found to be insignificant, for essentially the same reasons.

Summing up, it may be said that in interstellar space the contribution of ambipolar diffusion due to the interstellar magnetic fields is probably not so small as to be insignificant where hydrogen is largely unionized, and locally it may even contribute quite effectively there to the dissipation of kinetic energy.

Next, we consider the acceleration of cosmic-ray 
TABLE I.

\begin{tabular}{lccccr}
\hline & $\mathrm{H}$ & $E_{\perp}$ & $E_{\mathrm{II}}$ & $E_{\text {tot }}$ \\
& \multicolumn{5}{c}{ Arbitrary units } \\
\hline Original situation & 1 & 2 & 1 & 3 \\
After rapida increase of $\mathrm{H}$ & 10 & 20 & 1 & 21 \\
After redistribution at constant $\mathrm{H}$ & 10 & 14 & 7 & 21 \\
$\begin{array}{l}\text { After rapida decrease of } \mathrm{H} \text { to its } \\
\quad \text { original value }\end{array}$ & 1 & 1.4 & 7 & 8.4 \\
After redistribution at constant $\mathrm{H}$ & 1 & 5.6 & 2.8 & 8.4 \\
\hline
\end{tabular}

- Rapid relative to the collision frequency but slow relative to the gyration frequency.

particles as a sink of energy. As discussed at the conference on cosmic radiation which began in Varenna last week, the storage of the cosmic radiation in the interstellar magnetic fields is probably not quite as effective as was believed around 1950. The observed abundance of $\mathrm{Li}, \mathrm{Be}$, and $\mathrm{B}$ in the primary cosmic radiation indicates a somewhat smaller age of these particles than would be expected in the case of ideal storage in the interstellar magnetic fields. Since the energy density of the cosmic radiation is $10^{-12} \mathrm{erg} \mathrm{cm}^{-3}$, which apparently has to be replenished in say six million years, the energy required would then be not much less than $10^{-26} \mathrm{erg} \mathrm{cm}^{-3} \mathrm{sec}^{-1}$. If this energy is supplied by the interstellar gas in the way proposed by Fermi, it would constitute a quite important sink of energy.

We do not discuss this aspect of the theory of cosmic radiation as such, but discuss only one necessary condition which must be fulfilled for the Fermi mechanism to be operative in interstellar magnetic fields. This condition requires the magnetic field to change appreciably along a distance of the order of the gyration radius of the cosmic-ray particles, which is $\gtrsim 10^{12} \mathrm{~cm}$ for $\mathrm{H}=10^{-5}$ gauss. Such abrupt changes of the magnetic field are likely to occur only in shock fronts. If their widths are of the order of the gyration radius of the ions of the interstellar gas as proposed at this symposium by Petschek and others, the conditions would be much more favorable for the Fermi mechanism to be operative in interstellar space, than with the more conventional assumption of a width of a few mean free paths. In $\mathrm{HI}$ regions the mean free path will be of the order of $10^{14} \cdots 10^{15} \mathrm{~cm}$ for a density of $10^{1} \mathrm{part} / \mathrm{cm}$. Thus only clouds with density $>10^{3} \mathrm{part} / \mathrm{cm}$ would allow a sensible part of cosmic radiation to be accelerated. In ordinary dense HII regions the mean free path would be of the order of the gyration radius of cosmic radiation or smaller. Hence in these regions the conditions seems to be fulfilled for all cosmic-ray energies of interest (cf. also the evidence for turbulence in HII regions discussed later this symposium). In dilute HII regions in or between spiral arms the mean free path is again large compared with the gyration radius of most cosmic-ray particles.

Summing up, it appears that provided the mean free path (and not the gyration radius) determines effec- tively the width of the shock fronts, the condition for the Fermi mechanism to be operative, discussed in this section, is fulfilled only in dense HII regions.

As our last topic we discuss a dissipation mechanism depending on redistribution of energy after periodic changes of the magnetic field strength. This mechanism has been discussed in another connection by one of us (A.S.) at the Venice meeting on Ionization Phenomena in Gases a fortnight ago. We first indicate briefly how it works and then show that it may operate quite effectively in the solar corona.

For this we propose to consider a plasma in a homogeneous magnetic field, the intensity of which is changed periodically with a frequency of the order of the collision frequency of the ions. The kinetic energy of the ions (and electrons) may be divided into the parts belonging to the motion parallel and perpendicular to the magnetic field, respectively. Denoting these parts by $E_{\|}, E_{1}$, and their sum by $E_{\text {tot }}$, the constancy of the magnetic moment associated with the spiraling motion between collisions implies that $E_{1}$ changes proportionately to the magnetic field strength until collisions redistribute the total energy according to the equilibrium ratio of $E_{\perp}$ to $E_{||}$-namely, 2:1. In an artificial example we show (see Table I) how periodic changes of the magnetic field increase $E_{\text {tot }}$, that is to say the thermal energy and the entropy. Increasing the magnetic field by a factor $10, E_{\perp}$ becomes 10 times the original value which we may take $=2$, and $E_{\text {tot }}$ becomes 21. Redistribution by collisions leaves $E_{\perp}$ to $E_{\|}$in proportion 14:7, which becomes $1.4: 7$ after reducing rapidly the magnetic field intensity to its original value. Thus, $E_{\text {tot }}$ is then 8.4 and $E_{1}$ and $E_{l 1}$, after redistribution by further collisions, 5.6 and 2.8 , respectively. Hence in each such cycle the total energy increases by a factor 2.8 .

This mechanism requires the time scale of the disturbances of the magnetic intensity to be of the order of the collision frequency or faster (but slower than the gyration frequency). The process converts the energy which is needed to maintain the variation with time of the magnetic field into heat. Since the change of the magnetic field is accompanied by a change of density of the plasma, it acts somewhat like an additional volume viscosity of a plasma. It seems to be of no particular importance in the interstellar gas under normal circumstances (except in shock fronts). Let us however consider the solar corona and assume that its energy balance is maintained in the way suggested by Schwarzschild and one of us (L.B.), that is to say by pressure waves originating from the solar granulation zone. The time scale of these pressure waves, which act at the same time on the local magnetic field, is of the order of $10^{-2} \mathrm{sec}^{-1}$ according to the observations of the solar granulation. At a level in the corona where the density is $10^{7}$ part $/ \mathrm{cm}$ or less, the collision frequency drops to $10^{-2} \mathrm{sec}^{-1}$ or less. It appears then that the 
condition in question is fulfilled and that the heating by the change of the magnetic intensity, as discussed above, may contribute effectively to the dissipation of the energy of these waves, though it is not suggested that they contribute more than the ordinary viscous phenomena.

\section{DISCUSSION}

M. P. SAVEDOFF, Rochester University, Rochester, New York: Yesterday, Parker pointed out that a magnetic field may inhibit collisions between the clouds in two dimensions while probably in the third dimension everything is free. I conclude that the kinetic energy of the clouds would go into hydromagnetic waves. If hydromagnetic waves are propagating through the $\mathrm{HI}$ regions, will they be efficiently dissipated by the ambipolar diffusion?

L. BIERMANN, Göttingen, Germany: My answer is contained in the figures given in the paper. Your question is then whether the energy going into hydromagnetic waves is merely dissipated by ambipolar diffusion. That may be true, but I think one should not distinguish really between the hydromagnetic waves and pressure waves. Every wave type which arises under these conditions is a mixed hydromagnetic and ordinary shock wave. The whole energy of both the magnetic field and the velocity field is dissipated mainly by the turbulent viscosity, which is assisted to some degree by ambipolar diffusion.

E. Schatzman, Institut d'Astrophysique, Paris, France: Ambipolar diffusion may be of great importance in the region separating an ordinary HI from an ordinary HII region, because there you have a much higher number of neutral atoms and not many less ions. Hence, in between an ordinary HII and HI region where the gas is not fully ionized and not fully neutral, you may have a dissipation possibly reaching $10^{-26}$ erg $\mathrm{cm}^{-3} \mathrm{sec}^{-1}$. That might be a very efficient way of heating matter.

L. BIERMANN : I think Schatzman's remark is quite important, and it is easy to use the formulas on the blackboard and to change the figures in such a way as to evaluate it numerically.

(A discussion in which Parker, Biermann, Schlüter, and van de Hulst took part, concerning the estimates of the energy input into the interstellar medium was taken up again in the general discussion of Wednesday. The remarks brought forward have been omitted here and the reader is referred to the summary given in Sec. III-Editors.)

M. P. SAVEDOFF : A little while ago I looked into this question of the energy input from stars using the actual radio astronomical data, which I will discuss later in the Symposium. An important point is that the stars are most efficient in putting energy into the interstellar medium when they occur in gas clouds of low density.

M. MIN NAERT, Sterrewacht Sonnenburg, Utrecht, Netherlands: Must we take into account somewhere in these considerations the report of Pickelner and Shklovsky yesterday, and the factor 25 by which they estimate the dissipation of energy to be decreased in the presence of a magnetic field? How far do these considerations apply to the outer parts of the galactic system, that is to the galactic halo? Is it possible that in the galactic halo we have these interactions between $\mathrm{HI}$ and HII regions? It does not seem very probable, since there you would expect to have all the matter ionized. In that case, there must be a mechanism of transfer of the energy input from the central parts of the galaxy to the outer parts of the galactic corona.

L. BIERMAN : To the second point, accepting that there is a galactic halo of fairly low density - say only $1 \%$ or less of the mean value for the disk-one would expect that this halo is continuously heated by pressure waves arising from the expanding HII regions. One might use the solar chromosphere and the solar corona as an example. The solar corona is believed to be heated by pressure waves coming from below and degenerating into shock waves because the densities become small. In an analogous way, one would expect that energy is flowing into the halo and will maintain it at rather high temperature. As far as this goes, I would conclude that the probability that it is ionized and very hot-I think Dr. Spitzer considers $10^{6}$ to be the temperature of the halo-looks very much more probable than a situation where part of the medium is un-ionized.

H. C. VAN DE HULST, Leiden Observatory, Leiden, Netherlands: I have a question for Biermann about the possible influence of the ambipolar diffusion on the distribution of temperatures we might expect in the neutral hydrogen clouds. If we accept the picture given by Kahn during the last Symposium, that most of the heating is done by separate collisions once in ten million years, then the clouds we look at at any moment have an enormous range of temperatures. If also the ambipolar diffusion would come in as a permanent source of heating, the distribution of temperatures might be more even, i.e., the extremes might be avoided. Have you looked into this problem?

L. BIERMAN : No, I have not compared this supply of energy for the temperature in the sense mentioned by van de Hulst. I think we could discuss it. 
R. N. THOMAS, National Bureau of Standards, Boulder, Colorado: Just one question which Minnaert raised and which is puzzling from the secretary's standpoint. Do I understand, from Parker's presentation yesterday, that his original conclusion of wanting to greatly decrease the amount of dissipation by the magnetic field should now be withdrawn, because it only operates in two dimensions and in the third dimension one has rapid dissipation?

E. N. PARKER, Enrico Fermi Institute for Nuclear Studies, Chicago, Illinois: I believe the magnetic field considerably decreases the over-all dissipation of energy by a factor of at least 2 or 3 . Less by a factor 10 if the dissipation is actually to fit Oort's input estimates.

R. N. Thomas: We have then two viewpoints. Parker, Pickelner, and Shklovsky suggest much less dissipation as a consequence of magnetic inhibition; Biermann and the rest want the old figure for dissipation. Thus, the major uncertainty is the energy dissipation. Is this a correct summary?

E. N. PARKER : No, we cannot quarrel with dissipation estimates until we settle the point about the energy input, then I will know how much dissipation we can tolerate.

R. N. THOMAS: This is a caricature of a theoretician's remark. You should be able to settle how much dissipation you expect, quite apart from how much you need. What your remark really means is that you are completely uncertain as to these magnetic inhibition effects, and want to see what is required in order to assess them.

M. P. SAVEDOFF : Seaton has implied that the observed temperatures of $125^{\circ}$, with current cross sections for the radiation of the energy, requires a strong dissipative mechanism of the order of $10^{-27}$ erg $\mathrm{cm}^{-3} \mathrm{sec}^{-1}$. This is needed to maintain an observed temperature of $125^{\circ}$, instead of an equilibrium temperature down around the $20^{\circ}$ that Spitzer and others have found.
E. N. PARKER : I think Savedoff correctly quoted Seaton on the point, but in a private discussion Seaton mentioned that the $10^{-27}$ is based on the assumption that every ten million years a cloud bumps another cloud and the temperature jumps to a few thousand degrees and then cools off during the next ten million years. He said if the energy input were somewhat more continuous so that you never had the extreme temperature but always were near $120^{\circ}$-and this is the point that van de Hulst raised about the uniformity of the temperature-the figure could be dropped to $10^{-28}$. Seaton will probably comment on this in his paper.

F. D. KAHN, Manchester University, Manchester England: I would just like to ask Biermann whether the nonuniform heating would still remain when ambipolar diffusion is important, depending on whether the magnetic field is highly strained by the motions which have taken place, or whether it is more or less even. You get more ambipolar diffusion when the matter is trying to get through the field and therefore more heating.

L. BIERMANN : That is correct. Whether the whole of the balance is simply that given here or not, what is causing ambipolar diffusion is the fact that the plasma is in a sense moved by the magnetic field; any inhomogeneity of the magnetic field causes a volume force on the plasma but not on the non-ionized particles, and that gives a sort of differential acceleration, causing the ambipolar diffusion.

E. SCHATZMAN : When you have a collision between an HI and HII cloud in the presence of a magnetic field, then you have a shock front and you also have an ambipolar diffusion at the limit of the two regions. What does this do for the equilibrium temperature between the HI and HII regions through the front?

L. BIERMANN : We have not considered this question in detail, but I would agree that when you look into the detailed temperature distribution one must certainly follow this line. 